UDC 519.872

PACS 07.05.Tp, 02.60.Pn, 02.70.Bf

DOI: $10.22363 / 2658-4670-2020-28-1-49-61$

\title{
Two-way communication retrial queue with unreliable server and multiple types of outgoing calls
}

\author{
Anatoly A. Nazarov, Svetlana V. Paul, Olga D. Lizyura \\ Institute of Applied Mathematics and Computer Science \\ National Research Tomsk State University \\ 36, Lenina St., Tomsk, 634050, Russian Federation
}

(received: March 25, 2020; accepted: April 10, 2020)

\begin{abstract}
Retrial queue under consideration is the model of call center operator switching between input and outgoing calls. Incoming calls form a Poisson point process. Upon arrival, an incoming call occupies the server for an exponentially distributed service time if the server is idle. If the server if busy, an incoming call joins the orbit to make a delay before the next attempt to take the server. The probability distribution of the length of delay is an exponential distribution. Otherwise, the server makes outgoing calls in its idle time. There are multiple types of outgoing calls in the system. Outgoing call rates are different for each type of outgoing call. Durations of different types of outgoing calls follow distinct exponential distributions. Unsteadiness is that the server crashes after an exponentially distributed time and needs recovery. The rates of breakdowns and restorations are different and depend on server state. Our contribution is to obtain the probability distribution of the number of calls in the orbit under high rate of making outgoing calls limit condition. Based on the obtained asymptotics, we have built the approximations of the probability distribution of the number of calls in the orbit.
\end{abstract}

Key words and phrases: retrial queue, Poisson process, unreliable server, twoway communication, outgoing calls, incoming calls, asymptotic analysis, Gaussian approximation

\section{Introduction}

Blended call centers are an efficient and productive implement in modern companies. In addition to the usual call center features they can provide both incoming and outgoing calls. In its idle time the server initiates an outgoing call. Such behaviour allows to increase the effectiveness of the system. To study the functioning of the system with likewise particularity retrial queues are commonly used [1]-[3].

In retrial queues the customers that find the server busy repeat their request for service after a random delay. The pool of waiting customers is

(C) Nazarov A. A., Paul S. V., Lizyura O.D., 2020 
called an orbit. The most extensive research of this type of queueing systems is given in monographs [4], [5]. Retrial queues are usually the models of telecommunication and random access systems and their modifications allow to describe various real systems.

In this paper, we consider retrial queue with two-way communication and unreliable server. Two-way communication arose as a modification of retrial queues for modeling call centers with mixed calls. The Markovian models with outgoing calls are researched by Artalejo and Phung-Duc [6], [7]. Twoway communication queues have a branch of modifications to concrete the performance such as: finite input source [8], server-orbit interaction [9], [10], call-back option [11], etc. In our case there are multiple types of outgoing calls in the system.

The unreliability is explained by the need to take into account possible interruptions in the operation of the server [12], [13]. In our definition the rates of breakdowns are different and depend on the server current state. To study the model we use asymptotic analysis method under high rates of making outgoing calls condition [14].

\section{Model description}

We consider single server retrial queue with multiple types of outgoing calls. Input process is a stationary Poisson process with rate $\lambda$. Incoming calls occupy the server for an exponentially distributed time with rate $\mu_{1}$. Calls that find the server busy join the orbit and repeat their attempt to take the server after an exponentially distributed delay with rate $\sigma$. In its idle time the server makes outgoing calls of type $n$ with rate $\alpha_{n}$ and provides the service for an exponentially distributed time with parameter $\mu_{n}$. For convenience, we number the types of outgoing calls from 2 to $N$. Unsteadiness of the system is a behaviour of the server that it crashes after an exponentially distributed time of functioning. The rate of breakdowns and restorations are distinguished and depend on the server state. Figure 1 depicts the structure of the system.

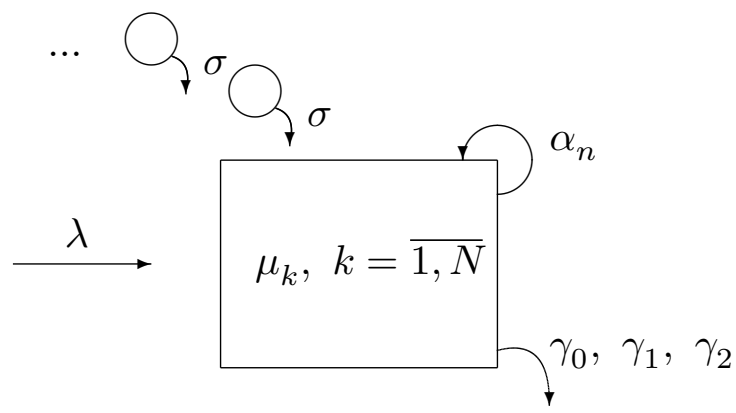

Figure 1. Single server retrial queue with unreliable server and multiple types of outgoing calls 
Let $k(t)$ denotes the state of the server at the time $t \geqslant 0$,

$$
k(t)= \begin{cases}0, & \text { if the server is idle, } \\ 1, & \text { if an incoming call is in service, } \\ n, & \text { if an outgoing call of type } n \text { is in service, } n=\overline{2, N} \\ N+1, & \text { if the server is in recovery state. }\end{cases}
$$

As the server breakdowns depend on its state we denote $\gamma_{0}$ is the rate of breakdowns in state $0, \gamma_{1}$ is the rate of breakdowns in state 1 and $\gamma_{2}$ is the rate of restorations. We assume that when the outgoing call is in service there is no breakdowns as the server calls the customer itself. If the customer is in service the breakdown interrupts the service and the customer joins the orbit.

Let $i(t)$ denotes the number of calls in the orbit at the moment $t \geqslant 0$. It is easy to see that two-dimensional process $\{i(t), k(t)\}$ is a continuous time Markov chain.

Let $P\{i(t)=i, k(t)=k\}=P_{k}(i, t)$ denotes the probability distribution of the process $\{i(t), k(t)\}$, then it is the solution of Kolmogorov's system of equations. We present the system in stationary regime

$$
\left\{\begin{array}{l}
-\left(\lambda+i \sigma+\sum_{n=2}^{N} \alpha_{n}+\gamma_{0}\right) P_{0}(i)+\sum_{k=1}^{N} \mu_{k} P_{k}(i)+\gamma_{2} P_{N+1}(i)=0, \\
-\left(\lambda+\mu_{1}+\gamma_{1}\right) P_{1}(i)+\lambda P_{1}(i-1)+\lambda P_{0}(i)+(i+1) \sigma P_{0}(i+1)=0, \\
-\left(\lambda+\mu_{n}\right) P_{n}(i)+\lambda P_{n}(i-1)+\alpha_{n} P_{0}(i)=0, \quad n=\overline{2, N} \\
-\left(\lambda+\gamma_{2}\right) P_{N+1}(i)+\lambda P_{N+1}(i-1)+\gamma_{0} P_{0}(i)+\gamma_{1} P_{1}(i-1)=0 .
\end{array}\right.
$$

Let $H_{k}(u)$ denotes the partial characteristic functions $H_{k}(u)=\sum_{i=0}^{\infty} e^{j u i} P_{k}(i)$, $k=\overline{0, N+1}$, where $j=\sqrt{-1}$. Multiplying equations of system (1) by $e^{j u i}$ and taking the sum over $i$ yields

$$
\left\{\begin{array}{l}
-\left(\lambda+\gamma_{0}+\sum_{n=2}^{N} \alpha_{n}\right) H_{0}(u)+j \sigma H_{0}^{\prime}(u)+ \\
\quad+\sum_{k=1}^{N} \mu_{k} H_{k}(u)+\gamma_{2} H_{N+1}(u)=0, \\
\left(\lambda\left(e^{j u}-1\right)-\mu_{1}-\gamma_{1}\right) H_{1}(u)+\lambda H_{0}(u)-j \sigma e^{-j u} H_{0}^{\prime}(u)=0, \\
\left(\lambda\left(e^{j u}-1\right)-\mu_{n}\right) H_{n}(u)+\alpha_{n} H_{0}(u)=0, \quad n=\overline{2, N}, \\
\left(\lambda\left(e^{j u}-1\right)-\gamma_{2}\right) H_{N+1}(u)+\gamma_{0} H_{0}(u)+\gamma_{1} e^{j u} H_{1}(u)=0, \\
j \sigma e^{-j u} H_{0}^{\prime}(u)+\left(\lambda+\gamma_{1}\right) H_{1}(u)+\lambda \sum_{n=2}^{N+1} H_{n}(u)=0,
\end{array}\right.
$$


where the last equation is an additional equation that we obtained by summing up the equations of the system.

The characteristic function $H(u)$ of the studied process can be expressed through the partial characteristic functions as follows

$$
H(u)=\sum_{k=0}^{N+1} H_{k}(u) .
$$

The main contribution of this research is the solution of the system (2) by using an asymptotic analysis method under high rate of outgoing calls limit condition.

\section{Asymptotic Analysis of the Model under the High Rate of Making Outgoing Calls}

In this section, we will investigate system (2) by asymptotic analysis method under the high rate of making outgoing calls limit condition. In particular, we prove that asymptotic characteristic function of the number of incoming calls in the system corresponds to Gaussian distribution.

To match the asymptotic condition we denote $\alpha_{n}=\alpha \nu_{n}$ in system (2)

$$
\left\{\begin{array}{l}
-\left(\lambda+\gamma_{0}+\alpha \sum_{n=2}^{N} \nu_{n}\right) H_{0}(u)+j \sigma H_{0}^{\prime}(u)+ \\
\quad+\sum_{k=1}^{N} \mu_{k} H_{k}(u)+\gamma_{2} H_{N+1}(u)=0, \\
\left(\lambda\left(e^{j u}-1\right)-\mu_{1}-\gamma_{1}\right) H_{1}(u)+\lambda H_{0}(u)-j \sigma e^{-j u} H_{0}^{\prime}(u)=0, \\
\left(\lambda\left(e^{j u}-1\right)-\mu_{n}\right) H_{n}(u)+\alpha \nu_{n} H_{0}(u)=0, \quad n=\overline{2, N}, \\
\left(\lambda\left(e^{j u}-1\right)-\gamma_{2}\right) H_{N+1}(u)+\gamma_{0} H_{0}(u)+\gamma_{1} e^{j u} H_{1}(u)=0, \\
j \sigma e^{-j u} H_{0}^{\prime}(u)+\left(\lambda+\gamma_{1}\right) H_{1}(u)+\lambda \sum_{n=2}^{N+1} H_{n}(u)=0 .
\end{array}\right.
$$

then the limit condition takes the form $\alpha \rightarrow \infty$.

\subsection{First Order Asymptotic}

We denote $\alpha=1 / \varepsilon$ in the system (3), and introduce the following notations

$$
u=\varepsilon w, \quad H_{0}(u)=\varepsilon F_{0}(w, \varepsilon), \quad H_{k}(u)=F_{k}(w, \varepsilon), \quad k=\overline{1, N+1},
$$

in order to get the following system 


$$
\left\{\begin{array}{c}
-\left(\lambda \varepsilon+\gamma_{0} \varepsilon+\sum_{n=2}^{N} \nu_{n}\right) F_{0}(w, \varepsilon)+j \sigma \frac{\partial F_{0}(w, \varepsilon)}{\partial w}+ \\
\quad+\sum_{k=1}^{N} \mu_{k} F_{k}(w, \varepsilon)+\gamma_{2} F_{N+1}(w, \varepsilon)=0 \\
\left(\lambda\left(e^{j w \varepsilon}-1\right)-\mu_{1}-\gamma_{1}\right) F_{1}(w, \varepsilon)+\lambda \varepsilon F_{0}(w, \varepsilon)- \\
\quad-j \sigma e^{-j w \varepsilon} \frac{\partial F_{0}(w, \varepsilon)}{\partial w}=0 \\
\left(\lambda\left(e^{j w \varepsilon}-1\right)-\mu_{n}\right) F_{n}(w, \varepsilon)+\nu_{n} F_{0}(w, \varepsilon)=0, n=\overline{2, N} \\
\left(\lambda\left(e^{j w \varepsilon}-1\right)-\gamma_{2}\right) F_{N+1}(w, \varepsilon)+\gamma_{0} \varepsilon F_{0}(w, \varepsilon)+\gamma_{1} e^{j w \varepsilon} F_{1}(w, \varepsilon)=0 \\
j \sigma e^{-j w \varepsilon} \frac{\partial F_{0}(w, \varepsilon)}{\partial w}+\left(\lambda+\gamma_{1}\right) F_{1}(w, \varepsilon)+\lambda \sum_{n=2}^{N+1} F_{n}(w, \varepsilon)=0 .
\end{array}\right.
$$

Theorem 1. Suppose $i(t)$ is the number of incoming calls in the aforementioned system of the stationary $M / M / 1$ retrial queue with several types of outgoing calls, then the (5) holds

$$
\lim _{\alpha \rightarrow \infty} E e^{j w \frac{i(t)}{\alpha}}=e^{j w \kappa_{1}},
$$

where

$$
\kappa_{1}=\frac{\lambda}{\sigma} \frac{\gamma_{2}\left(\mu_{1}+\gamma_{1}\right)}{\mu_{1} \gamma_{2}-\lambda\left(\gamma_{1}+\gamma_{2}\right)} \sum_{n=2}^{N} \frac{\nu_{n}}{\mu_{n}}
$$

Proof. Considering the limit as $\varepsilon \rightarrow 0$ in the system (4), then we will get

$$
\left\{\begin{array}{l}
-\sum_{n=2}^{N} \nu_{n} F_{0}(w)+j \sigma F_{0}^{\prime}(w)+\sum_{k=1}^{N} \mu_{k} F_{k}(w)+\gamma_{2} F_{N+1}(w)=0 \\
-\left(\mu_{1}+\gamma_{1}\right) F_{1}(w)-j \sigma F_{0}^{\prime}(w)=0 \\
-\mu_{n} F_{n}(w)+\nu_{n} F_{0}(w)=0, n=2, N \\
-\gamma_{2} F_{N+1}(w)+\gamma_{1} F_{1}(w)=0 \\
j \sigma F_{0}^{\prime}(w)+\left(\lambda+\gamma_{1}\right) F_{1}(w)+\lambda \sum_{n=2}^{N+1} F_{n}(w)=0 .
\end{array}\right.
$$

We propose to seek the solution of the system (7) in the following form

$$
F_{k}(w)=r_{k} \Phi(w), \quad k=\overline{0, N+1},
$$

where $r_{k}$ is the stationary probability distribution of the system states, then, dividing the equations by $\Phi(w)$, we obtain the system in the following form 


$$
\left\{\begin{array}{l}
-r_{0} \sum_{n=2}^{N} \nu_{n}+j \sigma r_{0} \frac{\Phi^{\prime}(w)}{\Phi(w)}+\sum_{k=1}^{N} \mu_{k} r_{k}+\gamma_{2} r_{N+1}=0 \\
-\left(\mu_{1}+\gamma_{1}\right) r_{1}-j \sigma r_{0} \frac{\Phi^{\prime}(w)}{\Phi(w)}=0 \\
-\mu_{n} r_{n}+\nu_{n} r_{0}=0, \quad n=\overline{2, N} \\
-\gamma_{2} r_{N+1}+\gamma_{1} r_{1}=0, \\
j \sigma r_{0} \frac{\Phi^{\prime}(w)}{\Phi(w)}+\left(\lambda+\gamma_{1}\right) r_{1}+\lambda \sum_{n=2}^{N+1} r_{n}=0 .
\end{array}\right.
$$

As the relation $\frac{\Phi^{\prime}(w)}{\Phi(w)}$ doesn't depend on $w$, the characteristic function $\Phi(w)$ can be expressed as $\Phi(w)=\exp \left\{j w \kappa_{1}\right\}$, which coincides with (5). Thus, we rewrite the system

$$
\left\{\begin{array}{l}
-\left(\sum_{n=2}^{N} \nu_{n}+\sigma \kappa_{1}\right) r_{0}+\sum_{k=1}^{N} \mu_{k} r_{k}+\gamma_{2} r_{N+1}=0 \\
-\left(\mu_{1}+\gamma_{1}\right) r_{1}+\sigma \kappa_{1} r_{0}=0 \\
-\mu_{n} r_{n}+\nu_{n} r_{0}=0 \\
-\gamma_{2} r_{N+1}+\gamma_{1} r_{1}=0, n=\overline{2, N} \\
-\sigma \kappa_{1} r_{0}+\left(\lambda+\gamma_{1}\right) r_{1}+\lambda \sum_{n=2}^{N+1} r_{n}=0
\end{array}\right.
$$

To obtain the values of $r_{k}$ we use second, third and fourth equations of the system with normalization condition

$$
\left\{\begin{array}{l}
r_{1}=\frac{\sigma \kappa_{1}}{\mu_{1}+\gamma_{1}} r_{0} \\
r_{n}=\frac{\nu_{n}}{\mu_{n}} r_{0}, n=\overline{2, N} \\
r_{N+1}=\frac{\gamma_{1}}{\gamma_{2}} r_{1}=\frac{\sigma \kappa_{1} \gamma_{1}}{\gamma_{2}\left(\mu_{1}+\gamma_{1}\right)} r_{0} \\
\sum_{k=1}^{N+1} r_{k}=\frac{\sigma \kappa_{1}}{\mu_{1}+\gamma_{1}} r_{0}+\sum_{n=2}^{N} \frac{\nu_{n}}{\mu_{n}} r_{0}+\frac{\sigma \kappa_{1} \gamma_{1}}{\gamma_{2}\left(\mu_{1}+\gamma_{1}\right)} r_{0}=1
\end{array}\right.
$$

From the last equality we obtain the value of $r_{0}$

$$
r_{0}=\left(\frac{\sigma \kappa_{1}\left(\gamma_{1}+\gamma_{2}\right)}{\gamma_{2}\left(\mu_{1}+\gamma_{1}\right)}+\sum_{n=2}^{N} \frac{\nu_{n}}{\mu_{n}}\right)^{-1}
$$


using which we can write the values $r_{k}$ as follows

$$
\left\{\begin{array}{l}
r_{1}=\frac{\sigma \kappa_{1}}{\mu_{1}+\gamma_{1}}\left(\frac{\sigma \kappa_{1}\left(\gamma_{1}+\gamma_{2}\right)}{\gamma_{2}\left(\mu_{1}+\gamma_{1}\right)}+\sum_{n=2}^{N} \frac{\nu_{n}}{\mu_{n}}\right)^{-1}, \\
r_{n}=\frac{\nu_{n}}{\mu_{n}}\left(\frac{\sigma \kappa_{1}\left(\gamma_{1}+\gamma_{2}\right)}{\gamma_{2}\left(\mu_{1}+\gamma_{1}\right)}+\sum_{k=2}^{N} \frac{\nu_{k}}{\mu_{k}}\right)^{-1}, \quad n=\overline{2, N} \\
r_{N+1}=\frac{\sigma \kappa_{1} \gamma_{1}}{\gamma_{2}\left(\mu_{1}+\gamma_{1}\right)}\left(\frac{\sigma \kappa_{1}\left(\gamma_{1}+\gamma_{2}\right)}{\gamma_{2}\left(\mu_{1}+\gamma_{1}\right)}+\sum_{n=2}^{N} \frac{\nu_{n}}{\mu_{n}}\right)^{-1},
\end{array}\right.
$$

The explicit value of $\kappa_{1}$ we obtain from the last equation of the system (10) using the equations (11)

$$
\kappa_{1}=\frac{\left(\lambda+\gamma_{1}\right) r_{1}+\lambda \sum_{n=2}^{N+1} r_{n}}{\sigma r_{0}}=\frac{\lambda}{\sigma} \frac{\gamma_{2}\left(\mu_{1}+\gamma_{1}\right)}{\mu_{1} \gamma_{2}-\lambda\left(\gamma_{1}+\gamma_{2}\right)} \sum_{n=2}^{N} \frac{\nu_{n}}{\mu_{n}},
$$

which coincides with (6).

\subsection{Second Order Asymptotic}

We introduce the following notations in the system (3)

$$
H_{k}(u)=e^{j u \alpha \kappa_{1}} H_{k}^{(2)}(u),
$$

to obtain the system of equations (16)

$$
\left\{\begin{array}{c}
-\left(\lambda+\gamma_{0}+\sigma \alpha \kappa_{1}+\alpha \sum_{n=2}^{N} \nu_{n}\right) H_{0}^{(2)}(u)+j \sigma \frac{d H_{0}^{(2)}(u)}{d u}+ \\
\quad+\sum_{k=1}^{N} \mu_{k} H_{k}^{(2)}(u)+\gamma_{2} H_{N+1}^{(2)}(u)=0, \\
\left(\lambda\left(e^{j u}-1\right)-\mu_{1}-\gamma_{1}\right) H_{1}^{(2)}(u)+\left(\lambda+\sigma \alpha \kappa_{1}\right) H_{0}^{(2)}(u)- \\
-j \sigma e^{-j u} \frac{d H_{0}^{(2)}(u)}{d u}=0, \\
\left(\lambda\left(e^{j u}-1\right)-\mu_{n}\right) H_{n}^{(2)}(u)+\alpha \nu_{n} H_{0}^{(2)}(u)=0, \quad n=\overline{2, N}, \\
\left(\lambda\left(e^{j u}-1\right)-\gamma_{2}\right) H_{N+1}^{(2)}(u)+\gamma_{0} H_{0}^{(2)}(u)+\gamma_{1} e^{j u} H_{1}^{(2)}(u)=0, \\
j \sigma e^{-j u} \frac{d H_{0}^{(2)}(u)}{d u}-\sigma \alpha \kappa_{1} e^{-j u} H_{0}^{(2)}(u)+\left(\lambda+\gamma_{1}\right) H_{1}^{(2)}(u)+ \\
+\lambda \sum_{n=2}^{N+1} H_{n}^{(2)}(u)=0 .
\end{array}\right.
$$


Denoting $\alpha=1 / \varepsilon^{2}$, and introducing the following notations in the system (16)

$$
\begin{gathered}
u=w \varepsilon \\
H_{0}^{(2)}(u)=\varepsilon^{2} F_{0}^{(2)}(w, \varepsilon), \quad H_{k}^{(2)}(u)=F_{k}^{(2)}(w, \varepsilon), \quad k=\overline{1, N+1}
\end{gathered}
$$

we obtain

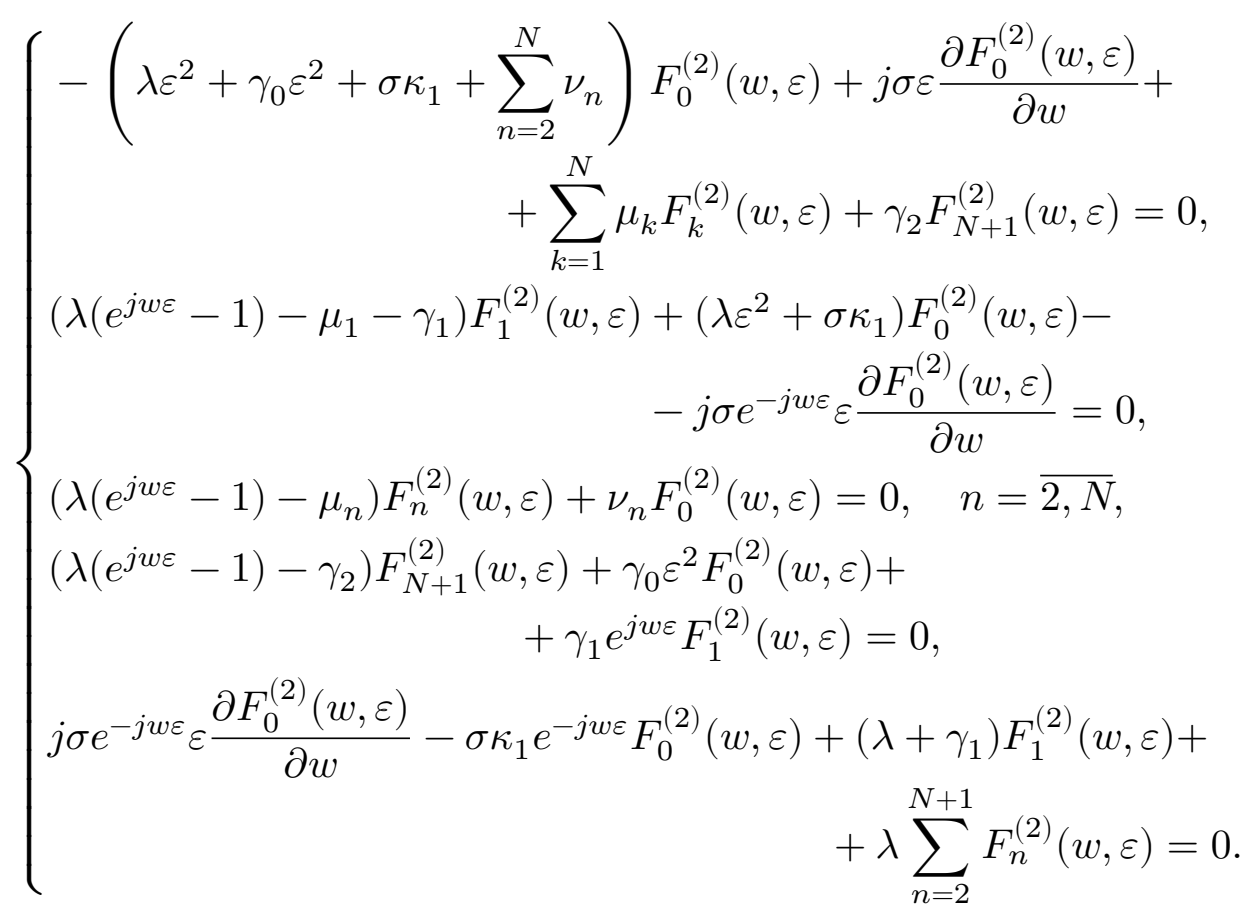

Theorem 2. In the context of Theorem 1 the following equation is true

$$
\lim _{\alpha \rightarrow \infty} E \exp \left\{j w \frac{\frac{i(t)}{\alpha}-\kappa_{1}}{\sqrt{\alpha}}\right\}=\exp \left\{\frac{(j w)^{2}}{2} \kappa_{2}\right\},
$$

where

$$
\begin{aligned}
\kappa_{2}= & \frac{\gamma_{2}\left(\mu_{1}+\gamma_{1}\right)}{\mu_{1} \gamma_{2}-\lambda \gamma_{2}-\lambda \gamma_{1}} \times \\
& \quad \times\left(\kappa_{1}+\lambda \kappa_{1} \frac{\gamma_{1}\left(\gamma_{1}+\gamma_{2}+\mu_{1}\right)\left(\lambda+\gamma_{2}\right)+\lambda \gamma_{2}^{2}}{\gamma_{2}^{2}\left(\mu_{1}+\gamma_{1}\right)^{2}}+\frac{\lambda^{2}}{\sigma} \sum_{n=2}^{N} \frac{\nu_{n}}{\mu_{n}^{2}}\right) .
\end{aligned}
$$

Proof. To solve the system of equations (18) we present the functions $F_{k}^{(2)}(w, \varepsilon)$ in the form

$$
F_{k}^{(2)}(w, \varepsilon)=\Phi_{2}(w)\left\{r_{k}+j w \varepsilon f_{k}\right\}+o\left(\varepsilon^{2}\right), k=\overline{0, N+1},
$$

here $r_{k}$ is the probability distribution of the server state obtained in Theorem 1. 
Substituting (21) into (18), making some simple conversions and taking (10) into account in the limit by $\varepsilon \rightarrow 0$, we obtain the system (22)

$$
\left\{\begin{array}{l}
\sigma \frac{\Phi_{2}^{\prime}(w)}{w \Phi(w)} r_{0}-\left(\sigma \kappa_{1}+\sum_{n=2}^{N} \nu_{n}\right) f_{0}+\sum_{k=1}^{N} \mu_{k} f_{k}+\gamma_{2} f_{N+1}=0 \\
-\sigma \frac{\Phi_{2}^{\prime}(w)}{w \Phi_{2}(w)} r_{0}+\lambda r_{1}-\left(\mu_{1}+\gamma_{1}\right) f_{1}+\sigma \kappa_{1} f_{0}=0 \\
\lambda r_{n}-\mu_{n} f_{n}+\nu_{n} f_{0}=0, \quad n=\overline{2, N} \\
\gamma_{1} r_{1}+\lambda r_{N+1}-\gamma_{2} f_{N+1}+\gamma_{1} f_{1}=0 \\
\sigma \frac{\Phi_{2}^{\prime}(w)}{w \Phi_{2}(w)} r_{0}+\sigma \kappa_{1} r_{0}-\sigma \kappa_{1} f_{0}+\left(\lambda+\gamma_{1}\right) f_{1}+\lambda \sum_{n=2}^{N+1} f_{n}=0 .
\end{array}\right.
$$

These equations imply that the relation $\frac{\Phi_{2}^{\prime}(w)}{w \Phi_{2}(w)}$ doesn't depend on $w$, thus the function $\Phi_{2}(w)$ is given in the following form

$$
\Phi_{2}(w)=\exp \left\{\frac{(j w)^{2}}{2} \kappa_{2}\right\}
$$

which coincides with (19).

We have $\frac{\Phi_{2}^{\prime}(w)}{w \Phi_{2}(w)}=-\kappa_{2}$ and then we obtain the system

$$
\left\{\begin{array}{l}
-\left(\sigma \kappa_{1}+\sum_{n=2}^{N} \nu_{n}\right) f_{0}+\sum_{k=1}^{N} \mu_{k} f_{k}+\gamma_{2} f_{N+1}=\sigma \kappa_{2} r_{0} \\
-\left(\mu_{1}+\gamma_{1}\right) f_{1}+\sigma \kappa_{1} f_{0}=-\sigma \kappa_{2} r_{0}-\lambda r_{1} \\
-\mu_{n} f_{n}+\nu_{n} f_{0}=-\lambda r_{n}, n=\overline{2, N} \\
-\gamma_{2} f_{N+1}+\gamma_{1} f_{1}=-\gamma_{1} r_{1}-\lambda r_{N+1} \\
-\sigma \kappa_{1} f_{0}+\left(\lambda+\gamma_{1}\right) f_{1}+\lambda \sum_{n=2}^{N+1} f_{n}=\sigma \kappa_{2} r_{0}-\sigma \kappa_{1} r_{0}
\end{array}\right.
$$

The system of equations (23) is heterogeneous form of the system (10), thus we represent the values $f_{k}$ in the form of

$$
f_{k}\left(\kappa_{2}\right)=C r_{k}+g_{k}\left(\kappa_{2}\right),
$$

and substitute (24) into (23). According to the (10) obtained in Theorem 1, the terms containing the constant $C$ are destroyed. We get system of equations 


$$
\left\{\begin{array}{l}
-\left(\sigma \kappa_{1}+\sum_{n=2}^{N} \nu_{n}\right) g_{0}+\sum_{k=1}^{N} \mu_{k} g_{k}+\gamma_{2} g_{N+1}=\sigma \kappa_{2} r_{0}, g_{k}=g_{k}\left(\kappa_{2}\right) \\
-\left(\mu_{1}+\gamma_{1}\right) g_{1}+\sigma \kappa_{1} g_{0}=-\sigma \kappa_{2} r_{0}-\lambda r_{1} \\
-\mu_{n} g_{n}+\nu_{n} g_{0}=-\lambda r_{n}, \quad n=\overline{2, N} \\
-\gamma_{2} g_{N+1}+\gamma_{1} g_{1}=-\gamma_{1} r_{1}-\lambda r_{N+1} \\
-\sigma \kappa_{1} g_{0}+\left(\lambda+\gamma_{1}\right) g_{1}+\lambda \sum_{n=2}^{N+1} g_{n}=\sigma \kappa_{2} r_{0}-\sigma \kappa_{1} r_{0}
\end{array}\right.
$$

From the second, third and fourth equations of the system (25) we have the following equalities

$$
\begin{gathered}
g_{1}=\frac{\sigma \kappa_{1}}{\mu_{1}+\gamma_{1}} g_{0}+\frac{\sigma \kappa_{2}}{\mu_{1}+\gamma_{1}} r_{0}+\frac{\lambda}{\mu_{1}+\gamma_{1}} r_{1}, \quad g_{n}=\frac{\nu_{n}}{\mu_{n}} g_{0}+\frac{\lambda}{\mu_{n}} r_{n}, \quad n=\overline{2, N} \\
g_{N+1}=\frac{\gamma_{1}}{\gamma_{2}} r_{1}+\frac{\gamma_{1}}{\gamma_{2}} g_{1}+\frac{\lambda}{\gamma_{2}} r_{N+1}= \\
=\frac{\sigma \kappa_{1} \gamma_{1}}{\gamma_{2}\left(\mu_{1}+\gamma_{1}\right)} g_{0}+\frac{\sigma \kappa_{2} \gamma_{1}}{\gamma_{2}\left(\mu_{1}+\gamma_{1}\right)} r_{0}+\frac{\gamma_{1}}{\gamma_{2}}\left(1+\frac{\lambda}{\mu_{1}+\gamma_{1}}\right) r_{1}+\frac{\lambda}{\gamma_{2}} r_{N+1} .
\end{gathered}
$$

Substituting these equalities into the last equation of the system (25) we get

$$
\begin{aligned}
& \left(-\sigma \kappa_{1}+\frac{\sigma \kappa_{1}\left(\lambda+\gamma_{1}\right)}{\mu_{1}+\gamma_{1}}+\lambda \sum_{n=2}^{N} \frac{\nu_{n}}{\mu_{n}}+\frac{\lambda \sigma \kappa_{1} \gamma_{1}}{\gamma_{2}\left(\mu_{1}+\gamma_{1}\right)}\right) g_{0}+ \\
& \quad+\left(\frac{\sigma \kappa_{2}\left(\lambda+\gamma_{1}\right)}{\mu_{1}+\gamma_{1}}+\frac{\lambda \sigma \kappa_{2} \gamma_{1}}{\gamma_{2}\left(\mu_{1}+\gamma_{1}\right)}-\sigma \kappa_{2}+\sigma \kappa_{1}\right) r_{0}+ \\
& +\left(\frac{\lambda\left(\lambda+\gamma_{1}\right)}{\mu_{1}+\gamma_{1}}+\frac{\lambda \gamma_{1}}{\gamma_{2}}\left(1+\frac{\lambda}{\mu_{1}+\gamma_{1}}\right)\right) r_{1}+\lambda \sum_{n=2}^{N} \frac{\lambda}{\mu_{n}} r_{n}+\frac{\lambda^{2}}{\gamma_{2}} r_{N+1}=0
\end{aligned}
$$

As the coefficient at $g_{0}$ is zero we can rewrite this equation to obtain the explicit expression of $\kappa_{2}$ which coincides with (20):

$$
\begin{aligned}
\kappa_{2}=\frac{\gamma_{2}\left(\mu_{1}+\gamma_{1}\right)}{\mu_{1} \gamma_{2}-\lambda \gamma_{2}-\lambda \gamma_{1}} \times & \\
& \times\left(\kappa_{1}+\lambda \kappa_{1} \frac{\gamma_{1}\left(\gamma_{1}+\gamma_{2}+\mu_{1}\right)\left(\lambda+\gamma_{2}\right)+\lambda \gamma_{2}^{2}}{\gamma_{2}^{2}\left(\mu_{1}+\gamma_{1}\right)^{2}}+\frac{\lambda^{2}}{\sigma} \sum_{n=2}^{N} \frac{\nu_{n}}{\mu_{n}^{2}}\right),
\end{aligned}
$$

To express the characteristic function $H(u)$ of the number of customers in the orbit we use the notations (15) and (17)

$$
H(u)=\exp \left\{j u \alpha \kappa_{1}+\left((j u)^{2} / 2\right) \alpha \kappa_{2}\right\} .
$$


Thus, in Theorem 2 we have obtained the variance $\kappa_{2} \alpha$ of a number of calls in the orbit in prelimit situation of $\alpha \rightarrow \infty$. The asymptotic probability distribution of the process $i(t)$ is Gaussian.

\section{Conclusions}

In this paper, we have considered the Markovian retrial queue with multiple types of outgoing calls and unreliable server. Using the asymptotic analysis method we have shown that the asymptotic behaviour of the number of customers in the orbit coincides with Gaussian distribution and in the limit condition $\alpha \rightarrow \infty$, which means high rates of making outgoing calls, we have the mean $\kappa_{1} \alpha$ and variance $\kappa_{2} \alpha$.

\section{Acknowledgments}

The publication has been funded by RFBR according to the research projects No. 19-41-703002.

\section{References}

[1] G. Koole and A. Mandelbaum, "Queueing models of call centers: An introduction," Annals of Operations Research, vol. 113, no. 1-4, pp. 4159, 2002. DOI: 10.1023/A: 1020949626017.

[2] S. Bhulai and G. Koole, "A queueing model for call blending in call centers," IEEE Transactions on Automatic Control, vol. 48, no. 8, pp. 14341438, 2003. DOI: 10.1109/TAC. 2003.815038.

[3] S. Aguir, F. Karaesmen, O. Z. Akşin, and F. Chauvet, "The impact of retrials on call center performance," OR Spectrum, vol. 26, no. 3, pp. 353-376, 2004.

[4] J. R. Artalejo and A. Gómez-Corral, Retrial Queueing Systems: A Computational Approach. Springer-Verlag Berlin Heidelberg, 2008.

[5] G. Falin and J. G. Templeton, Retrial queues. CRC Press, 1997, vol. 75.

[6] J. R. Artalejo and T. Phung-Duc, "Markovian retrial queues with two way communication," Journal of Industrial 83 Management Optimization, vol. 8, no. 4, pp. 781-806, 2012.

[7] J. R. Artalejo and T. Phung-Duc, "Single server retrial queues with two way communication," Applied Mathematical Modelling, vol. 37, no. 4, pp. 1811-1822, 2013. DOI: 10.1016/j .apm.2012.04.022.

[8] A. Nazarov, J. Sztrik, A. Kvach, and A. Kuki, "An Algorithmic Approach for the Analysis of Finite-Source M/GI/1 Retrial Queueing Systems with Collisions and Server Subject to Breakdowns and Repairs," in International Conference on Information Technologies and Mathematical Modelling, Springer, 2019, pp. 14-27. DOI: 10.1007/978-3-030-33388$1 \_2$.

[9] V. Dragieva and T. Phung-Duc, "Two-way communication M/M/1 retrial queue with server-orbit interaction," in Proceedings of the 11th International Conference on Queueing Theory and Network Applications, ACM, 2016, p. 11. DOI: 10.1145/3016032.3016049. 
[10] V. Dragieva and T. Phung-Duc, "Two-Way Communication M/M/1/1 Queue with Server-Orbit Interaction and Feedback of Outgoing Retrial Calls," in International Conference on Information Technologies and Mathematical Modelling, Springer, 2017, pp. 243-255. DOI: 10.1007/9783-319-68069-9_20.

[11] C. Kim, O. Dudina, A. Dudin, and S. Dudin, "Queueing system $\mathrm{MAP} / \mathrm{M} / \mathrm{N}$ as a model of call center with call-back option," in International Conference on Analytical and Stochastic Modeling Techniques and Applications, Springer, 2012, pp. 1-15. DOI: 10.1007/978-3-64230782-9_1.

[12] M. S. Kumar, A. Dadlani, and K. Kim, "Performance analysis of an unreliable M/G/1 retrial queue with two-way communication," Operational Research, pp. 1-14, 2018. DOI: 10.1007/s12351-018-0417-y.

[13] S. Paul and T. Phung-Duc, "Retrial Queueing Model with Two-Way Communication, Unreliable Server and Resume of Interrupted Call for Cognitive Radio Networks," in Information Technologies and Mathematical Modelling. Queueing Theory and Applications, Springer, 2018, pp. 213-224. DOI: 10.1007/978-3-319-97595-5_17.

[14] A. A. Nazarov, S. Paul, and I. Gudkova, "Asymptotic analysis of Markovian retrial queue with two-way communication under low rate of retrials condition," in Proceedings 31st European Conference on Modelling and Simulation, 2017, pp. 678-693. DOI: 10.7148/2017-0687.

\section{For citation:}

A. A. Nazarov, S. V. Paul, O. D. Lizyura, Two-way communication retrial queue with unreliable server and multiple types of outgoing calls, Discrete and Continuous Models and Applied Computational Science 28 (1) (2020) 49-61. DOI: $10.22363 / 2658-4670-2020-28-1-49-61$.

\section{Information about the authors:}

Nazarov, Anatoly A. (Russian Federation) - Doctor of Technical Sciences, head of Department of Probability Theory and Mathematical Statistics of National Research Tomsk State University (e-mail: nazarov.tsu@gmail.com, phone: +7(3822)529599, ORCID: https://orcid.org/0000-0002-2091-6011, ResearcherID: O-5862-2014, Scopus Author ID: 7201780364)

Paul, Svetlana V. (Russian Federation) - Candidate of Physical and Mathematical Sciences, Assistant Professor of Department of Probability Theory and Mathematical Statistics of National Research Tomsk State University (e-mail: paulsv82@mail.ru, phone: +7(3822)529599, ORCID: https://orcid.org/0000-0002-3681-0676, ResearcherID: E-7860-2017, Scopus Author ID: 57031144200)

Lizyura, Olga. D. (Russian Federation) - Master's Degree Student of Institute of Applied Mathematics and Computer Science, National Research Tomsk State University (e-mail: oliztsu@mail.ru, phone: +7(999)4996743, ORCID: https://orcid.org/0000-0003-4463-4876, ResearcherID: G-7896-2019, Scopus Author ID: 57212169399) 
УДК 519.872

PACS 07.05.Tp, 02.60.Pn, 02.70.Bf

DOI: $10.22363 / 2658-4670-2020-28-1-49-61$

\title{
RQ-система с ненадёжным прибором и разнотипными вызываемыми заявками
}

\author{
А. А. Назаров, С. В. Пауль, О. Д. Лизюра \\ Кафедра теории вероятностей и математической статистики \\ Наииональный исследовательский Томский государственный университет \\ ул. Ленина, д. 36, Томск, 634050, Россия
}

В статье RQ-система с разнотипными вызываемыми заявками рассматривается как модель оператора call-центра. Входящие звонки образуют простейший поток. В момент поступления заявка из потока занимает прибор для обслуживания, если он свободен. Распределение вероятностей длительностей обслуживания является экспоненциальным. Если прибор занят, поступившая заявка отправляется на орбиту, где осуществляет задержку случайной длительности, распределённой по экспоненциальному закону, после чего снова пытается занять прибор для обслуживания. С другой стороны, когда прибор свободен, он вызывает заявки извне. В системе есть несколько типов вызываемых заявок. Интенсивности вызывания различны для разных типов вызываемых заявок. Длительности обслуживания вызываемых заявок разных типов являются экспоненциальными случайными величинами с различными параметрами. Ненадёжность прибора характеризуется выходом из строя на период времени, длительность которого распределена экспоненциально. Интенсивности выхода из строя и восстановления прибора различны и зависят от состояния прибора. Целью исследования является получение стационарного распределения вероятностей числа заявок на орбите методом асимптотического анализа в предельном условии высокой интенсивности вызывания заявок. На основе полученного асимптотического распределения построена аппроксимация допредельного распределения вероятностей числа заявок на орбите в рассматриваемой RQ-системе.

Ключевые слова: RQ-система, простейший поток, ненадёжный прибор, вызываемые заявки, метод асимптотического анализа, гауссовская аппроксимация 